\title{
Ultrafast Demagnetization Measurements Using Extreme Ultraviolet Light: Comparison of Electronic and Magnetic Contributions
}

\author{
Chan La-O-Vorakiat, ${ }^{*}$ Emrah Turgut, Carson A. Teale, Henry C. Kapteyn, and Margaret M. Murnane \\ Department of Physics and JILA, and NSF Engineering Research Center in Extreme Ultraviolet Science and Technology, University of \\ Colorado and NIST, Boulder, Colorado 80309, USA \\ Stefan Mathias ${ }^{\dagger}$ and Martin Aeschlimann \\ University of Kaiserslautern and Research Center OPTIMAS, 67663 Kaiserslautern, Germany \\ Claus M. Schneider \\ Peter Grünberg Institute, PGI-6, Research Center Jülich, 52425, Jülich, Germany \\ Justin M. Shaw, Hans T. Nembach, and T. J. Silva \\ Electromagnetics Division, National Institute of Standards and Technology, Boulder, Colorado 80305, USA
}

(Received 26 October 2011; published 23 January 2012)

\begin{abstract}
Ultrashort pulses of extreme ultraviolet light from high-harmonic generation are a new tool for probing coupled charge, spin, and phonon dynamics with element specificity, attosecond pump-probe synchronization, and time resolution of a few femtoseconds in a tabletop apparatus. In this paper, we address an important question in magneto-optics that has implications for understanding magnetism on the fastest time scales: Is the signal from the transverse magneto-optical Kerr effect at the $\mathrm{M}_{2,3}$ edges of a magnetic material purely magnetic or is it perturbed by nonmagnetic artifacts? Our measurements demonstrate conclusively that transverse magneto-optical Kerr measurements at the $\mathrm{M}_{2,3}$ edges sensitively probe the magnetic state, with almost negligible contributions from the transient variation of the refractive index by the nonequilibrium hot-electron distribution. In addition, we compare pump-probe demagnetization dynamics measured by both high harmonics and conventional visible-wavelength magneto-optics and find that the measured demagnetization times are in agreement.
\end{abstract}

DOI: 10.1103/PhysRevX.2.011005

Subject Areas: Magnetism, Optics

A challenging research topic of current interest in material science is to understand what correlated dynamics governs laser-induced demagnetization on ultrafast time scales [1-3]. In such experiments, an intense ultrashort femtosecond laser pulse is used to quench the magnetization within a few hundred femtoseconds. The resultant time scale for demagnetization is then inferred by monitoring the reduction in magnetic contrast using different magnetooptical probes. Fundamentally, demagnetization dynamics is governed by nonequilibrium interactions between electrons, spins, and phonons. The fundamental microscopic processes involved in these interactions are not well understood and are currently under intense debate [4-10].

Ever since the first pioneering work of femtosecond laser-induced demagnetization, there has existed a longstanding debate about the presence of nonmagnetic

\footnotetext{
*Corresponding author. Chan.La-o-vorakiat@Colorado.EDU

Corresponding author. smathias@physik.uni-kl.de

Published by the American Physical Society under the terms of the Creative Commons Attribution 3.0 License. Further distribution of this work must maintain attribution to the author(s) and the published article's title, journal citation, and DOI.
}

artifacts in the nominally magnetic signal induced by a femtosecond pump pulse [11-17]. In general, two physical mechanisms could give rise to nonmagnetic artifacts in a pump-probe experiment: state blocking, and the generation of nonequilibrium electron distributions. State blocking, or dichroic bleaching, is the transient saturation of an optical transition by the pump pulse. When pumping and probing the magnetic material with light pulses of the same wavelength and polarization, the pump pulse can saturate the same transition access by the probe pulse. Hence, stateblocking artifacts can for the most part be suppressed by using different pump and probe wavelengths.

A second optical artifact can be introduced by the transient hot-electron distribution generated in the sample by the pump pulse, which strongly and transiently modifies the occupation density of the valence states just above the Fermi level. Both nonmagnetic artifacts can be mostly canceled by measuring the magneto-optical contrast before and after the magnetization direction (the magnetic asymmetry, $A=\frac{I_{+}-I_{-}}{I_{+}+I_{-}}$, discussed below) is reversed. However, the remaining part of the artifact can alter the magnetic asymmetry via the transient change of the refractive index. This artifact influences all known time-resolved magneticprobe techniques (e.g., the magneto-optical Kerr effect, surface second-harmonic generation, $\mathrm{x}$-ray magnetic circular 
dichroism, and spin-polarized photoelectron spectroscopy) $[14,16]$.

Specifically, in the visible region of the spectrum, the magneto-optical Kerr effect (MOKE) is used to monitor the magnetic state by measuring the magnetization-induced polarization and/or the ellipticity change of reflected light. However, the observed magneto-optical signal may contain nonmagnetic artifacts $[11,14]$ that originate from a modification of the optical reflectivity on the time scale of a few hundred femtoseconds by the distorted density of unoccupied states. Optical probing of the transient magnetic state has another limitation: Given the relatively small photon energy of visible light $(h \nu \approx 1.5 \mathrm{eV})$, the measured MOKE signal represents collective excitations from all the constituent atoms, and any element-specific information is missing. Recently, ultrashort (about $100 \mathrm{fs}$ ) soft $\mathrm{x}$ rays from synchrotron sources have been used for element-specific probing of ultrafast magnetization dynamics by measuring $\mathrm{x}$-ray magnetic circular dichroism (XMCD) $[18,19]$. In XMCD, the X-ray absorption of a magnetic sample depends on the helicity (left- and righthanded) of a circularly polarized x-ray beam relative to the magnetization direction.

In a complementary approach, we recently demonstrated that tabletop high-harmonic-generation (HHG) light sources could be used as an element-selective probe of magnetization dynamics on ultrafast time scales. Using a sub-10-femtosecond extreme-ultraviolet (XUV) beam that spans the $\mathbf{M}_{2,3}$ absorption edges (i.e., electronic transitions between the $3 p$ core levels and $3 d$ valance levels), we simultaneously probed the demagnetization dynamics of $\mathrm{Fe}$ and $\mathrm{Ni}$ in permalloy [20]. In our experimental geometry, we measured the magnetic state using the transverse magneto-optical Kerr effect (T-MOKE), where a compo- nent of the reflected light at the $\mathrm{M}_{2,3}$ edges is proportional to the magnetization perpendicular to the plane of incidence. For each time delay between the laser pump pulses and the XUV probe pulses, we measured the normalized T-MOKE magnetic asymmetry, defined as the difference in sample reflectivity as the static magnetization direction is reversed with an externally applied magnetic field.

Since our probe of magnetization dynamics with XUV light relies on reflectivity, it is important to verify that the measured T-MOKE response at ultrafast time scales is predominantly of magnetic origin. In this paper, we demonstrate that the $\mathrm{M}_{2,3}$-edge T-MOKE signal in a timeresolved experiment is essentially free of transient artifacts that are not magnetic in origin. We detect a very small component of the signal that presumably originates from the generation of hot electrons and phonons in response to the pump pulse. This nonmagnetic component is 2 orders of magnitude smaller than the observed demagnetization signal. Having validated $\mathrm{M}_{2,3}$-edge T-MOKE for its magnetic sensitivity, we also present a systematic study of the pump-fluence dependence (pump-pulse energy per unit area) of demagnetization dynamics in Ni. These measurements agree very well with previous experimental results that employed conventional longitudinal magneto-optic Kerr effect (L-MOKE) in the visible range, as well as theoretical calculations [4].

In our experiment, we produce XUV radiation from HHG by focusing $90 \%$ of an amplified, femtosecond pulsed laser output (780-nm wavelength, $3-\mathrm{kHz}$ repetition rate, at $2 \mathrm{~mJ}$ per pulse) into a capillary waveguide filled with either Ne or Ar (Fig. 1). The duration of the XUV pulses is less than $10 \mathrm{fs}$. The output flux of XUV harmonics is maximized by varying the gas pressure in the waveguide to phase match the high-harmonic upcoversion process

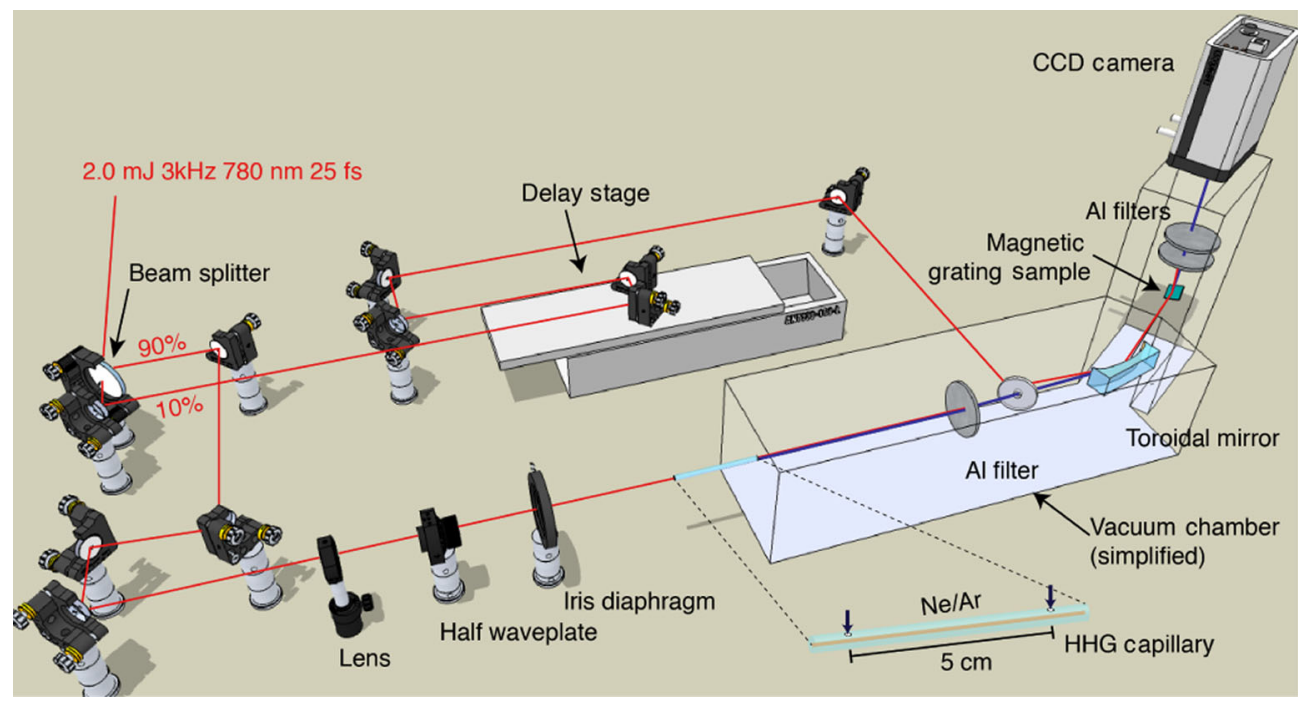

FIG. 1. Experimental setup. The magnetic grating sample is excited by a 25 -fs pump pulse and then probed by a time-delayed HHG probe pulse. To spectrally resolve the XUV spectra, the grating sample diffracts the probe beam that is detected by use of a CCD camera. The optical models are from Ref. [23]. 
$[21,22]$. The XUV beams have photon energies spanning $35-72 \mathrm{eV}$ in Ne, and $39-48 \mathrm{eV}$ in Ar. (The cutoff at $72 \mathrm{eV}$ is due to the absorption edge of the $\mathrm{Al}$ filters that are used to block the laser light copropagating with the probe HHG beam.) In the T-MOKE geometry, the polarizationdependent reflected intensity of the XUV beam is measured [24] as follows:

$I_{ \pm}^{p}=I_{0}\left|\frac{n \cos \theta_{i}-\cos \theta_{t}}{n \cos \theta_{i}+\cos \theta_{t}} \pm \frac{2 \sin \theta_{i} \cos \theta_{i}}{n^{2}\left(n \cos \theta_{i}+\cos \theta_{t}\right)^{2}} \epsilon_{x y}\right|^{2}$ for $p$-polarized incident light,

$I^{s}=I_{0}\left|\frac{\cos \theta_{i}-n \cos \theta_{t}}{\cos \theta_{i}+n \cos \theta_{t}}\right|^{2}$ for $s$-polarized incident light,

where $I_{0}$ is the incident beam intensity, $n$ is the refractive index of material, $\theta_{i}$ is the angle of incidence, $\theta_{t}=$ $\sin ^{-1}\left(\sin \theta_{i} / n\right)$ is the refractive angle, the plus-minus sign ( \pm ) depends on the direction of magnetization, and $\epsilon_{x y}$ is the magnetization-dependent off-diagonal element of the dielectric tensor. The first term inside the absolute square of Eq. (1) is the nonmagnetic Fresnel coefficient for the sample reflectivity in the case of $p$-polarized incident light. According to Eq. (2), the sample reflectivity for $s$-polarized incident light is always independent of the magnetization. In contrast, the reflectivity for $p$-polarized light depends on the magnetic part via the dielectric magneto-optical constant $\epsilon_{x y}$, which is linearly proportional to the magnetization component perpendicular to the plane of incidence. Here, we consider the change of the reflectivity up to the first order in the magneto-optical constant, since the magneto-optical constants are usually small numbers (around $10^{-3}$ ) [24].

To extract the magnetic signal from the reflected intensity of the $p$-polarized light, we calculate the asymmetry parameter $(A)$, which is the difference in reflected intensity from the sample before and after the magnetization reversal normalized by the sum of the intensities:

$$
\begin{aligned}
A & =\frac{I_{+}^{p}-I_{\underline{p}}^{p}}{I_{+}^{p}+I_{\underline{p}}^{p}} \cong 2 \operatorname{Re}\left[\frac{\sin \left(2 \theta_{i}\right) \epsilon_{x y}}{n^{4} \cos ^{2} \theta_{i}-n^{2}+\sin ^{2} \theta_{i}}\right] \\
& =2 \operatorname{Re}\left[\frac{\sin \left(2 \theta_{i}\right) \epsilon_{x y}}{\left(n^{2}-1\right)\left[n^{2}-\sin ^{2} \theta_{i}\left(n^{2}+1\right)\right]}\right] \\
& \cong \frac{\sin \left(2 \theta_{i}\right)}{1-2 \cos ^{2} \theta_{i}}\left(\frac{\delta \operatorname{Re}\left[\epsilon_{x y}\right]+\beta \operatorname{Im}\left[\epsilon_{x y}\right]}{\delta^{2}+\beta^{2}}\right) .
\end{aligned}
$$

In the first step, we assume that the Fresnel coefficient is large compared to the magneto-optical term, and, in the last step, we substitute the complex representation of the refractive index, $n=1-\delta+i \beta$, where $\delta$ and $\beta$ are small numbers. These equations give four important properties of the asymmetry: First, from the second line of Eq. (3), the asymmetry is maximized at the Brewster's angle near $45^{\circ}$ as has been previously derived in Ref. [25]. Second, the last line of Eq. (3) suggests that the T-MOKE magnetic asymmetry is resonantly enhanced with $A \propto \operatorname{Im}\left[\epsilon_{x y}\right] / \beta$, where $\delta$ approaches zero near the $\mathrm{M}_{2,3}$ edges. Third, the asymmetry is also a function of the refractive index $(n)$, so that any index change induced by the pump laser pulse could possibly introduce an artifact to the measured magnetic dynamics. We will later show that this effect is negligible compared to the T-MOKE magnetic signal in the XUV regime. Finally, the asymmetry is independent of the probe intensity provided that the intensity remains unchanged during a complete cycle of magnetic reversal. In practice, fluctuations of the HHG intensity may occur during the reversal cycle. We reduce this intensityfluctuation noise by switching the magnetization at a frequency of $1 \mathrm{~Hz}$ by use of an external magnetic field of $\pm 32 \mathrm{kA} / \mathrm{m}$ ( $\pm 400 \mathrm{Oe})$, which is sufficient to saturate the magnetization of our polycrystalline $\mathrm{Ni}$ samples.

The measured T-MOKE asymmetry is wavelength dependent because of the dispersive nature of the refractive index $n(\omega)$ and off-diagonal magnetic component of the dielectric tensor $\epsilon_{x y}(\omega)$ in the XUV range. In particular, $\epsilon_{x y}(\omega)$ is resonantly enhanced at the M edge in transition metals $[25,26]$. Thus, it is very important that the reflectivity is measured as a function of photon energy. To achieve this, we incorporate the magnetic samples into a simple spectrometer by fabricating the samples into diffraction gratings by a straightforward optical lithography/ liftoff process. For the work described here, two samples are used: The first sample consists of alternating stripes of $\mathrm{Ni}$ and $\mathrm{Fe}$ with a $2-\mu \mathrm{m}$ period and a $10-\mathrm{nm}$ thickness [Fig. 2(a)]. The sample is also composed of a Ta seed layer $(3 \mathrm{~nm})$ for the adhesion of $\mathrm{Ni}$ and Fe stripes, and a Ta cap layer $(2.5 \mathrm{~nm})$ on top of the Fe stripes only, in order to prevent oxidation. The second sample consists of purely $\mathrm{Ni}$ stripes with the same physical dimensions as in the first sample. The photon energy is resolved by capturing the diffraction pattern on a charge-coupled-device (CCD) camera with $45^{\circ}$ angle-of-incidence geometry on the sample to maximize the asymmetry (Fig. 1). The diffracted harmonics are somewhat broadened and overlapping because the diffracted harmonics at the CCD camera are slightly out of focus. By reversing the magnetic-field direction, we observe the asymmetry in reflection at the photon energies near the $\mathrm{M}_{2,3}$ edges of $\mathrm{Fe}$ and $\mathrm{Ni}$ [Fig. 2(b)]. Two 200-nm Al filters are used to block the 780-nm pump laser light from saturating the CCD camera.

To extract the demagnetization dynamics, we first select a harmonic with photon energy $h \nu=66.2 \pm 0.2 \mathrm{eV}$, which is close to the $\mathrm{Ni} \mathrm{M}_{2,3}$ edges at 66.2 and $68.0 \mathrm{eV}$, respectively, and we then calculate the asymmetry as a function of pump-probe time delay. Time zero, corresponding to overlap of the pump and probe pulses, is determined by using a second-harmonic crystal mounted at the sample position. The accuracy of the time-zero determination is within \pm 10 fs. We repeat this measurement for varying 


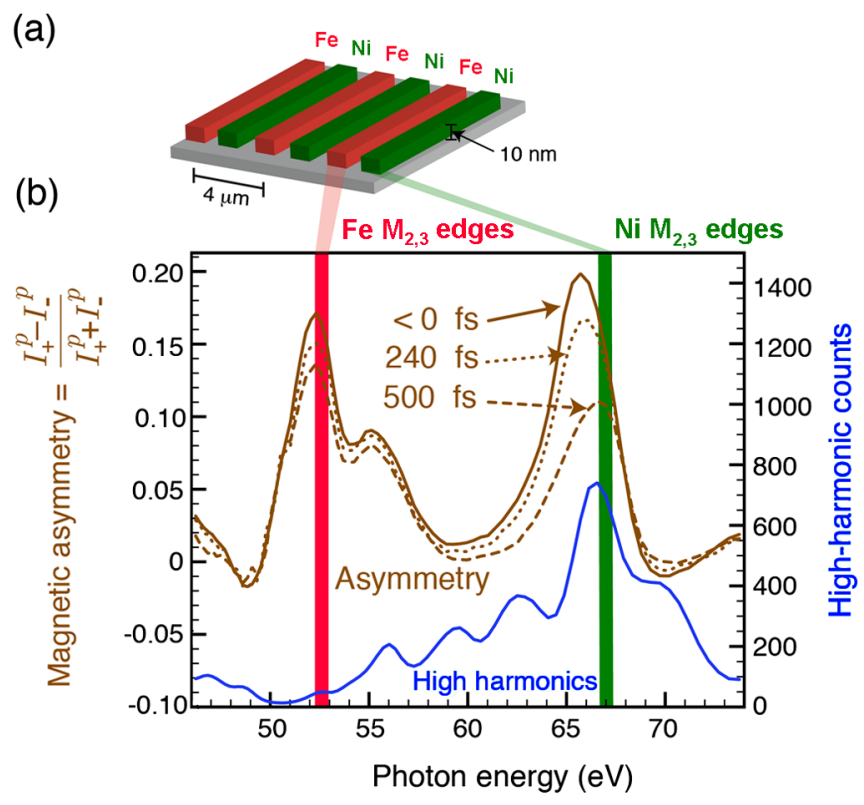

FIG. 2. (a) An alternating Ni-Fe grating sample geometry. (b) Typical HHG spectra near the $\mathrm{Fe}$ and $\mathrm{Ni} \mathrm{M}_{2,3}$ edges after reflection from the grating sample (blue curve). Magnetic asymmetry (brown curves) peaks at the expected locations of the $\mathrm{Ni}$ (green line) and $\mathrm{Fe}$ (red line) $\mathrm{M}_{2,3}$ edges for various delay times before and after the arrival of the demagnetization laser pulse at the sample.

pump fluences between 0.3 and $2.4 \mathrm{~mJ} / \mathrm{cm}^{2}$. The results of these measurements for both $p$-polarized (magnetic) and $s$-polarized (nonmagnetic) probes are shown in Fig. 3. The data for the $p$-polarized probe beam are fit to a phenomenological exponential function: $f\left(t, t_{0}\right)=$ $1-\Delta A\left(1-e^{\left(t-t_{0}\right) / \tau_{\mathrm{M}}}\right) e^{\left(t-t_{0}\right) / \tau_{\mathrm{R}}}$, where $\tau_{\mathrm{M}}$ is demagnetization time, $\tau_{\mathrm{R}}$ is magnetization recovery time, $\Delta A$ is demagnetization amplitude, and $t_{0}$ is the time zero of the dynamics, i.e., the delay time that results in maximum temporal overlap between pump and probe pulses. Both $\Delta A$ and $\tau_{\mathrm{M}}$ for the $p$-polarized pump are dependent on pump fluence, spanning from $\Delta A=5 \%$ to $50 \%$, while the demagnetization time varies from $\tau_{\mathrm{M}}=90 \pm 72$ to $150 \pm$ 19 fs. From these fits, we find that $\Delta A$ is linearly proportional to fluence. The signal-to-noise ratio is the smallest at low pump fluence, where the demagnetization amplitude approaches the noise level of $3.5 \%$.

To verify that the extracted magnetic-asymmetry dynamics corresponds to a purely magnetic signal, we isolate the nonmagnetic contribution to the reflectivity by using an $s$-polarized probe beam. For $s$-polarization, we cannot detect any asymmetry in the reflectivity as in the case of $p$-polarization, and the asymmetry is negligible over the entire time scan (Fig. 3). We also measure the nonmagnetic transient dynamics of the XUV $s$-polarization reflectivity induced by the pump laser pulse as a function of pumpprobe time delay (Fig. 4), for a pump fluence of $1 \mathrm{~mJ} / \mathrm{cm}^{2}$.

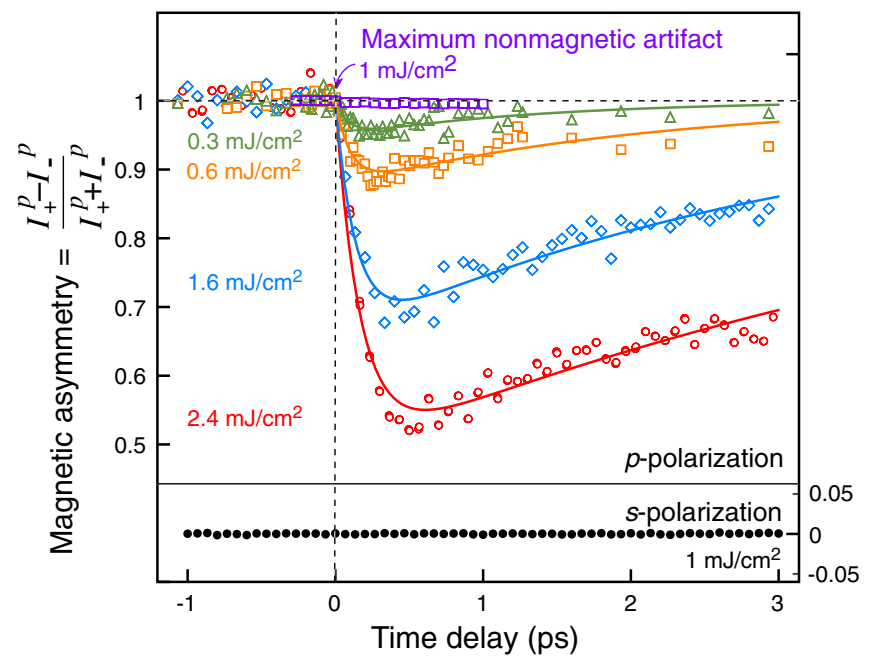

FIG. 3. Pump-fluence-dependent demagnetization dynamics of $\mathrm{Ni}$ for the Ni-Fe grating. The magnetic asymmetry in $\mathrm{Ni}$ is measured by selecting XUV photon energies near the $\mathrm{M}_{2,3}$ absorption edges of $\mathrm{Ni}(h \nu=66.2 \pm 0.2 \mathrm{eV})$. By varying the pump laser fluence from 0.3 to $2.4 \mathrm{~mJ} / \mathrm{cm}^{2}$, we can achieve up to $50 \%$ demagnetization. The demagnetization is linearly proportional to the pump fluence. From the reflectivity data in Fig. 4, we estimate the maximum nonmagnetic contribution to the asymmetry signal by considering the variation of the real and imaginary reflective index, $\delta$ or $\beta$ (purple open squares, which are on top of each other) during the first picosecond. The maximum nonmagnetic response is 2 orders of magnitude smaller than the demagnetization amplitude.

We employ harmonics both near the $\mathrm{M}_{2,3}$ edges of $\mathrm{Ni}$ $(h \nu=67.7 \pm 0.5 \mathrm{eV})$ and far below the edge $h \nu=$ $44.6 \pm 0.3 \mathrm{eV}$ ) in order to determine whether there is any resonant enhancement of reflectivity changes near the absorption edges. Both measurements at 44.6 and $67.7 \mathrm{eV}$ are performed in parallel by analyzing the HHG signal acquired in parallel. Therefore, the time axes of both data sets are identical.

The dependence of the measured reflectivity on time delay is presented in Fig. 4 . The $s$-polarization reflectivity at the $\mathrm{M}_{2,3}$ edges increases by only $0.2 \%$ during the initial $300 \mathrm{fs}$, which is the critical time scale for both demagnetization dynamics and the nonequilibrium hot-electron dynamics [14]. This fast change of the reflectivity results from the transient variation of the refractive index, since it is the only variable in Eq. (2), and the change is in accordance with transient-reflectivity measurements in the visible spectrum [27]. We can estimate the modification of the refractive index by calculating the upper limit for the change of the real and imaginary components of the refractive index $(n=\delta+i \beta)$ based on the measured transient change in the $s$-polarization reflectivity. If we assume that the $0.2 \%$ change in the $s$-polarization reflectivity is caused by variations of either $\delta$ or $\beta$ alone, which is a worst-case scenario, then numerical solution of Eq. (2) can be used to calculate that $\delta$ or $\beta$ would vary by no more than 


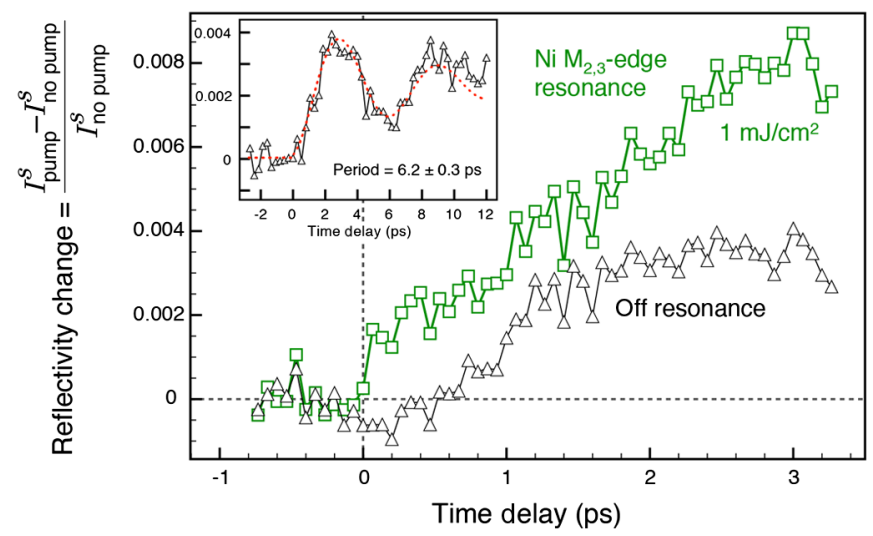

FIG. 4. Nonmagnetic reflectivity for a pump fluence of $1 \mathrm{~mJ} / \mathrm{cm}^{2}$, measured by use of an $s$-polarized XUV probe beam both near $(h \nu=67.7 \pm 0.5 \mathrm{eV}$, green squares) and far from ( $h \nu=44.6 \pm 0.3 \mathrm{eV}$, black triangles) the $\mathrm{Ni} \mathrm{M}_{2,3}$ edges. The maximum nonmagnetic artifact to the asymmetry can be inferred from this resonance reflectivity measurement (Fig. 3). We attribute the reflectivity dynamics at slower time scales in excess of a picosecond (inset) to the presence of a longitudinal acoustic wave that is launched by the absorption of pump-pulse energy by the periodic diffraction grating. By definition, the asymmetry parameter is unaffected by this phonon contribution because it results only in the modulation of the diffractiongrating efficiency.

$3 \%$ or $0.1 \%$, respectively. (Tabulated values for $\delta$ and $\beta$ as functions of XUV photon energy were used for this calculation [28].) We then calculate the expected transient change in the asymmetry from Eq. (3). We find that the measured time-dependent magnetic asymmetries can include an artifact of no more than $0.2 \%$ during the first $300 \mathrm{fs}$ of the demagnetization dynamics (Fig. 3). This potential artifact is 2 orders of magnitude smaller than the observed demagnetization amplitude of $20 \%$ as measured with the same pump fluence.

Another important feature of the data occurs at time scales well beyond a picosecond (Fig. 4): The reflectivity continues to increase 2.5-3 ps after the arrival of the pump pulse. We perform a fit to extract an exponential time constant of $3.27 \pm 1.37 \mathrm{ps}$. This time constant is about an order of magnitude slower than the demagnetization time (200-300 fs). This slow variation in reflectivity has, therefore, little effect on the measured ultrafast demagnetization dynamics. In addition, such a slow process cannot be due to nonequilibrium hot-electron dynamics because of fast electron-electron and electron-phonon scattering rates. Indeed, the process is not only slow but also oscillatory. (See the inset in Fig. 4.) To increase the XUV flux to resolve this oscillation, we extend the scan range to $12 \mathrm{ps}$ and switch the HHG medium to Ar. This fast rise in reflectivity is due to the thermal launching of a longitudinal acoustic wave in the grating structure. Our past work has shown that propagation of acoustic waves in grating structures causes the grating height to oscillate, which in turn modulates the grating efficiency and the reflectivity [29]. Hence, these longer-time-scale phononic signals are amplified in our detection scheme and contribute more strongly to the transient reflectivity changes than the shorter-time-scale nonequilibrium electronic signals. Fitting the data to an exponentially damped sinusoid yields a period of $6.3 \pm 0.3 \mathrm{ps}$. We use the tabulated value of the speed of the longitudinal wave of $\mathrm{Ni}(5489 \mathrm{~m} / \mathrm{s}), \mathrm{Fe}$ $(5960 \mathrm{~m} / \mathrm{s})$ [30], and Ta $(3956 \mathrm{~m} / \mathrm{s})$ [31] to calculate the round-trip time for the longitudinal acoustic wave to travel along the grating thickness $(10 \mathrm{~nm}$ of $\mathrm{Ni}$ and $\mathrm{Fe})$, the 2.5-nm Ta cap layer (on Fe stripes only), and the 3-nm $\mathrm{Ta}$ seed layer (of both $\mathrm{Fe}$ and Ni stripes). The estimates are $5.16 \mathrm{ps}$ for $\mathrm{Fe}$ and 6.16 for Ni. The average of the two numbers is in reasonable agreement with the measured period. The small discrepancy is probably the result of the height uncertainty of the sample, as the total thickness measured by an atomic-force microscope is about $2-3 \mathrm{~nm}$ larger than the ideal.

We reiterate that the magnetic-asymmetry measurement is not sensitive to such nonmagnetic variations in the sample reflectivity. In the case of phonon oscillations, the $p$-polarization reflectivity is simply modulated by the timedependent change in the grating efficiency. This timedependent change in grating efficiency can be represented by an additional scaling factor for the reflected intensities in Eqs. (1) and (2). Since the same factor appears in both numerator and denominator in the definition of the magnetic asymmetry in Eq. (3), it cancels out. This assumption is valid as long as the phononic spectrum does not cause a change in the electronic selection rules that might subsequently modify the refractive indexes. These phononic effects might subsequently modify the refractive indexes. However, from our experimental result (Fig. 3), we exclude such effects to be significant for the extracted magneticasymmetry signal. Moreover, phonon dynamics evolve on a time scales slower than the ultrafast demagnetization dynamics in the $3 d$ ferromagnetic systems considered here.

Our data show that T-MOKE measurements by use of XUV radiation at the $\mathrm{M}_{2,3}$ edges of Ni are sensitive predominantly to the ultrafast magnetic response. For this reason, we are well positioned to benchmark conventional, visible-wavelength L-MOKE measurements of ultrafast demagnetization times against our artifact-free T-MOKE results obtained at XUV energies. In Fig. 5, we present the demagnetization times measured by use of XUV $\mathrm{M}_{2,3}$-edges T-MOKE from both sample types (Ni-Fe grating and $\mathrm{Ni}$ grating) as a function of demagnetization amplitude $\Delta A$, and we compare our results with those in Ref. [4] that were obtained by using $1.5 \mathrm{eV}$ (pump) and $3 \mathrm{eV}$ (probe) light. The theoretical calculation that is also shown in Fig. 5 is derived from a microscopic threetemperature model based on Elliot-Yafet-type spin-flip scattering processes, as presented in Ref. [4]. It is clear from Fig. 5 that the XUV T-MOKE data are in excellent 


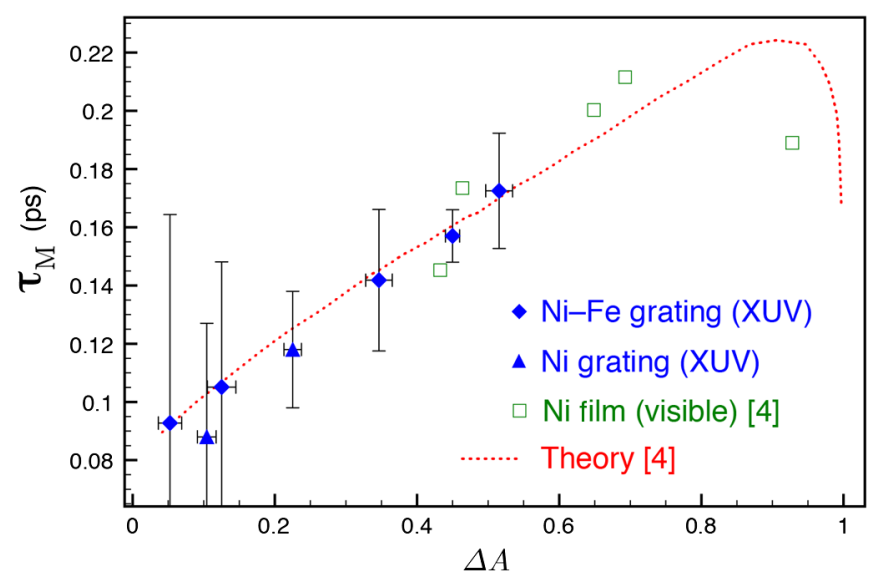

FIG. 5. Comparison of demagnetization times measured by use of XUV light ( $h \nu=66.2 \pm 0.2 \mathrm{eV}$, blue shapes) from the present study and by use of visible light $(h \nu=3.1 \mathrm{eV}$, open green squares) from Ref. [4]. The blue diamonds represent the data from alternating $\mathrm{Ni}-\mathrm{Fe}$ grating samples, while the blue triangles represent the data from $\mathrm{Ni}$ grating samples. All experimental data are in good agreement with the theoretical prediction (dotted red line) [4]. The error bars are calculated from the confidence bounds of the fitting coefficients.

agreement with both the theoretical and the experimental L-MOKE results obtained using visible light—despite the vastly different probe energies, different electronic ground states, sample structures, and experimental geometries. In the limit of zero fluence, our data linearly extrapolate to demagnetization times of $78.4 \pm 9 \mathrm{fs}$, in good agreement with previously presented data for $\mathrm{Ni}$ in the limit of a very weak pump fluence $(\Delta A \approx 0.02)$ [32]. In addition, the similarity of the data for both of our sample types implies that this measurement method is robust against detailed variations of the sample structure.

Finally, we would like to compare our data with that obtained using femtosecond XMCD [18]. The demagnetization time measured with XMCD agrees with the T-MOKE-XUV and visible-MOKE data, when the larger error bar of the XMCD data is taken into account (120 \pm $70 \mathrm{fs}$ when the demagnetization amplitude $\Delta A$ is about 0.6). The two techniques are complementary to each other, and each has its own advantages. T-MOKE is sensitive to the transverse component of magnetization with respect to the plane of incidence, while XMCD is sensitive to the component of magnetization that is parallel to the propagation vector of light. XUV can be implemented in both reflection and transmission modes, whereas soft-x-ray measurements are best performed in transmission mode. Sum rules can be used to distinguish spin and orbital magnetic moment with XMCD data. However, T-MOKE with HHG currently yields superior time resolution and allows for a tabletop setup.

In conclusion, we have validated T-MOKE detection at the $\mathrm{M}_{2,3}$ edges of $\mathrm{Ni}$ as an artifact-free probe of ultrafast magnetization dynamics. By comparing data acquired with $s$ - and $p$-polarized probe pulses, we have shown that the magnetic-asymmetry signal obtained with XUV photons in the T-MOKE geometry is predominantly of magnetic origin. Any nonmagnetic contribution to the asymmetry parameter is small $(0.2 \%)$ in comparison to the amplitude of demagnetization $(20 \%)$ at the same fluence. For longer time scales ( $>1 \mathrm{ps})$, we detect an oscillatory modulation of reflectivity, resulting from acoustic wave generation. This slow modulation of the reflectivity cannot contribute to a magnetic-asymmetry measurement. Having established that T-MOKE at XUV near the $\mathrm{M}_{2,3}$ edges of $\mathrm{Ni}$ is insensitive to both refractive-index changes and reflectivity changes of nonmagnetic origin, we then compare our measured demagnetization times for $\mathrm{Ni}$ over a wide range of pump fluences with previously published experimental and theoretical results acquired with visible-wavelength L-MOKE. We find that the results obtained by both measurement techniques are in excellent agreement. The results presented here are foundational for future XUV studies of ultrafast magnetization dynamics.

We would like to thank the authors of Ref. [4] for allowing us to reproduce their data. The authors also gratefully acknowledge funding from the US Department of Energy Office of Basic Energy Sciences, Contract No. DE-FG02-09ER46652. S. M. was supported by the European Community's FP7 under the Marie Curie International Outgoing Fellowship No. GA 253316.

[1] E. Beaurepaire, J. C. Merle, A. Daunois, and J. Y. Bigot, Ultrafast Spin Dynamics in Ferromagnetic Nickel, Phys. Rev. Lett. 76, 4250 (1996).

[2] B. Koopmans, in Spin Dynamics in Confined Magnetic Strucures II, edited by B. Hillebrands and K. Ounadjela (Springer-Verlag, Berlin, 2003).

[3] M. Cinchetti, M. Sanchez Albaneda, D. Hoffmann, T. Roth, J.-P Wuestenberg, M. Krauss, O. Andreyev, H. C. Schneider, M. Bauer, and M. Aeschlimann, Spin-Flip Processes and Ultrafast Magnetization Dynamics in Co: Unifying the Microscopic and Macroscopic View of Femtosecond Magnetism, Phys. Rev. Lett. 97, 177201 (2006).

[4] B. Koopmans, G. Malinowski, F. Dalla Longa, D. Steiauf, M. Faehnle, T. Roth, M. Cinchetti, and M. Aeschlimann, Explaining the Paradoxical Diversity of Ultrafast LaserInduced Demagnetization, Nature Mater. 9, 259 (2010).

[5] M. Krauss, T. Roth, S. Alebrand, D. Steil, M. Cinchetti, M. Aeschlimann, and H.C. Schneider, Ultrafast Demagnetization of Ferromagnetic Transition Metals: The Role of the Coulomb Interaction, Phys. Rev. B 80, 180407 (2009).

[6] G.P. Zhang and W. Hübner, Laser-Induced Ultrafast Demagnetization in Ferromagnetic Metals, Phys. Rev. Lett. 85, 3025 (2000). 
[7] J. Y. Bigot, M. Vomir, and E. Beaurepaire, Coherent Ultrafast Magnetism Induced by Femtosecond Laser Pulses, Nature Phys. 5, 515 (2009).

[8] M. Battiato, K. Carva, and P. M. Oppeneer. Superdiffusive Spin Transport as a Mechanism of Ultrafast Demagnetization, Phys. Rev. Lett. 105, 027203 (2010).

[9] A. B. Schmidt M. Pickel, M. Donath, P. Buczek, A. Ernst, V.P. Zhukov, P. M. Echenique, L. M. Sandratskii, E. V. Chulkov, and M. Weinelt, Ultrafast Magnon Generation in an Fe Film on Cu(100), Phys. Rev. Lett. 105, 197401 (2010).

[10] B. Y. Mueller, T. Roth, M. Cinchetti, M. Aeschlimann, and B. Rethfeld, Driving Force of Ultrafast Magnetization Dynamics, New J. Phys. 13, 123010 (2011).

[11] B. Koopmans, M. van Kampen, J. T. Kohlhepp, and W. J. M. de Jonge, Ultrafast Magneto-Optics in Nickel: Magnetism or Optics?, Phys. Rev. Lett. 85, 844 (2000).

[12] L. Guidoni, E. Beaurepaire, and J. Y. Bigot, MagnetoOptics in the Ultrafast Regime: Thermalization of Spin Populations in Ferromagnetic Films, Phys. Rev. Lett. 89, 017401 (2002).

[13] J. Y. Bigot, L. Guidoni, E. Beaurepaire, and P. Saeta, Femtosecond Spectrotemporal Magneto-Optics, Phys. Rev. Lett. 93, 077401 (2004).

[14] P. M. Oppeneer and A. Liebsch, Ultrafast Demagnetization in Ni: Theory of Magneto-Optics for Non-Equilibrium Electron Distributions, J. Phys. Condens. Matter 16, 5519 (2004).

[15] G. P. Zhang, W. Hübner, G. Lefkidis, Y. Bai, and T. F. George, Paradigm of the Time-Resolved Magneto-Optical Kerr Effect for Femtosecond Magnetism, Nature Phys. 5, 499 (2009).

[16] K. Carva, D. Legut, and P. M Oppeneer, Influence of Laser-Excited Electron Distributions on the X-Ray Magnetic Circular Dichroism Spectra: Implications for Femtosecond Demagnetization in Ni, Europhys. Lett. 86, 57002 (2009).

[17] K. Carva, M. Battiato, and P. M. Oppeneer, Is the Controversy over Femtosecond Magneto-Optics Really Solved?, Nature Phys. 7, 665 (2011).

[18] C. Stamm, T. Kachel, N. Pontius, R. Mitzner, T. Quast, K. Holldack, S. Khan, C. Lupulescu, E. F. Aziz, M. Wietstruk, H. A. Dürr, and W. Eberhardt, Femtosecond Modification of Electron Localization and Transfer of Angular Momentum in Nickel, Nature Mater. 6, 740 (2007).

[19] I. Radu, K. Vahaplar, C. Stamm, T. Kachel, N. Pontius, H. A. Dürr, T. A. Ostler, J. Barker, R. F. L. Evans, R. W. Chantrell, A. Tsukamoto, A. Itoh, A. Kirilyuk, Th. Rasing, and A. V. Kimel, Transient Ferromagnetic-Like State Mediating Ultrafast Reversal of Antiferromagnetically Coupled Spins, Nature (London) 472, 205 (2011).

[20] C. La-O-Vorakiat, M. Siemens, M. M. Murnane, H. C. Kapteyn, S. Mathias, M. Aeschlimann, P. Grychtol, R. Adam, C. M. Schneider, J. M. Shaw, H. Nembach, and T. J. Silva, Ultrafast Demagnetization Dynamics at the $M$
Edges of Magnetic Elements Observed Using a Tabletop High-Harmonic Soft X-Ray Source, Phys. Rev. Lett. 103, 257402 (2009).

[21] T. Popmintchev, M.-C. Chen, P. Arpin, M. M. Murnane, and H. C. Kapteyn, The Attosecond Nonlinear Optics of Bright Coherent X-Ray Generation, Nature Photon. 4, 822 (2010).

[22] A. Rundquist, C. G. Durfee, Z.H. Chang, C. Herne, S. Backus, M. M. Murnane, and H.C. Kapteyn, PhaseMatched Generation of Coherent Soft X-Rays, Science 280, 1412 (1998).

[23] 3D Warehouse, http://sketchup.google.com/3dwarehouse/ search?uq=0774178905215056886102796\&scoring $=\mathrm{m}$. Mention of commercial products is for illustration only; it does not imply National Institute of Standards and Technology recommendation or endorsement, nor does it imply that the products illustrated are necessarily the best available for the purpose.

[24] H. Hochst, D. Rioux, D. Zhao, and D. L. Huber, Magnetic Linear Dichroism Effects in Reflection Spectroscopy: A Case Study at the Fe $\mathrm{M}_{2,3}$ Edge, J. Appl. Phys. 81, 7584 (1997).

[25] M. Hecker, P. M. Oppeneer, S. Valencia, H.-Ch. Mertins, and C.M. Schneider, Soft X-Ray Magnetic Reflection Spectroscopy at the $3 p$ Absorption Edges of Thin Fe Films, J. Electron Spectrosc. Relat. Phenom. 144-147, 881 (2005).

[26] M. Pretorius, J. Friedrich, A. Ranck, M. Schroeder, J. Voss, V. Wedemeier, D. Spanke, D. Knabben, I. Rozhko, H. Ohldag, F. U. Hillebrecht, and E. Kisker, Transverse Magneto-Optical Kerr Effect of Fe at the Fe $3 p$ Threshold, Phys. Rev. B 55, 14133 (1997).

[27] J. Hohlfeld, S.-S. Wellershoff, J. Güdde, U. Conrad, V. Jähnke, and E. Matthias, Electron and Lattice Dynamics Following Optical Excitation of Metals, Chem. Phys. 251, 237 (2000).

[28] E. Gullikson, X-Ray Interactions with Matter, http://henke .lbl.gov/optical_constants/.

[29] R. I. Tobey, M.E. Siemens, O. Cohen, M. M. Murnane, H.C. Kapteyn, and K. Nelson, Ultrafast Extreme Ultraviolet Holography: Dynamic Monitoring of Surface Deformation, Opt. Lett. 32, 286 (2007).

[30] R. C. Weast, CRC Handbook of Chemistry and Physics (CRC Press, Boca Raton, Florida, 1986), 67th ed., p. E-43.

[31] N. Soga, Comparison of Measured and Predicted Bulk Moduli of Tantalum and Tungsten at High Temperatures, J. Appl. Phys. 37, 3416 (1966). The speed of the longitudinal wave $\left(\nu_{l}\right)$ is calculated from the reported elastic constant $\left(c_{11}, 2602\right.$ kbar $)$, density $\left(\rho, 16.626 \mathrm{~g} / \mathrm{cm}^{3}\right)$ and the relation $\nu_{l}=\sqrt{\frac{c_{11}}{\rho}}$ : L. D. Landau and E. M. Lifshitz, Theory of Elasticity (Butterworth-Heinemann, Oxford, 1986), Vol. 7, 3rd ed..

[32] F. Dalla Longa, J. T. Kohlhepp, W. J. M. de Jonge, and B. Koopmans, Influence of Photon Angular Momentum on Ultrafast Demagnetization in Nickel, Phys. Rev. B 75, 224431 (2007). 